\title{
High temperature point sensor using tin-doped silica fiber gratings
}

\author{
G. Brambilla, H.H. Kee, V. Pruneri and T. P. Newson \\ Optoelectronics Research Centre, University of Southampton, Southampton, SO17 1BJ, UK
}

We describe the use of tin-doped silica fiber-grating sensors at high temperature. Results confirm the longterm thermal stability for a large temperature range. Measurements up to $\sim 800 \mathrm{oC}$ show a significant advantage over conventional fiber gratings.

\begin{abstract}
1. Introduction
Recently there has been intense research in the performance of miniature optical fiber thermometer probes for industrial and medical applications with minimal intrusion [1], because of the notable advantages in hostile environments, such as in the presence of high electric, magnetic or strong RF fields. One thermometry technique is to use the fluorescence emission from rare earth-doped and transition metal-doped fibers. The use of fluorescence in Cr:LiSAF resulted in a thermometer operating between a range of $0{ }^{\circ} \mathrm{C}$ to $100{ }^{\circ} \mathrm{C}$ [2], and recently with $\mathrm{Cr}: Y A G[3]$, and $\mathrm{Tm}$ [4] operating above $400{ }^{\circ} \mathrm{C}$. Another possible transducer for temperature measurements is based on the measurement of the change in reflected Bragg wavelength with temperature. However, conventional fiber grating thermometers written in germanosilicate fibers exhibit poor stability at high temperature, especially if hydrogen loaded [5]. In this paper, we show the enhanced thermal stability and excellent reliability of grating sensors written in tin-doped silica fibers (SS) up to a temperature of approximately $800{ }^{\circ} \mathrm{C}$. At this temperature all the other gratings have been completely erased.
\end{abstract}

\section{Experimental results}

The SS fiber was fabricated via modified chemical vapor deposition [6] and had NA 0.1 and cut-off wavelength $\lambda_{\mathrm{c}} \sim 1.3 \mu \mathrm{m}$. Gratings were written using a $\mathrm{KrF}$ excimer laser delivering $20 \mathrm{~ns}$ pulses at $20 \mathrm{~Hz}$. Pulse fluence was estimated to be $\sim 100 \mathrm{~mJ} / \mathrm{cm}^{2}$. Gratings have also been written in germanosilicate (SG), boro-germanosilicate (SGB) and hydrogen loaded telecom (THL) fibers. A grating written in tin-germanosilicate fiber was used as a reference. The gratings were spliced together and all of them except the reference have been placed in a furnace. Grating reflectivity was tested by launching light from a white light source and monitoring the reflected light with an optical spectrum analyzer via a coupler. The grating temperature was raised at a rate of $\sim 25^{\circ} \mathrm{C} / \mathrm{min}$, stabilized at working temperature for 4 hours and then decreased in steps to room temperature. Reflection spectra were recorded before each thermal step and at intervals of 15-60 mins at working temperature. Every cycle was repeated before increasing the working temperature in steps of $100^{\circ} \mathrm{C}$.

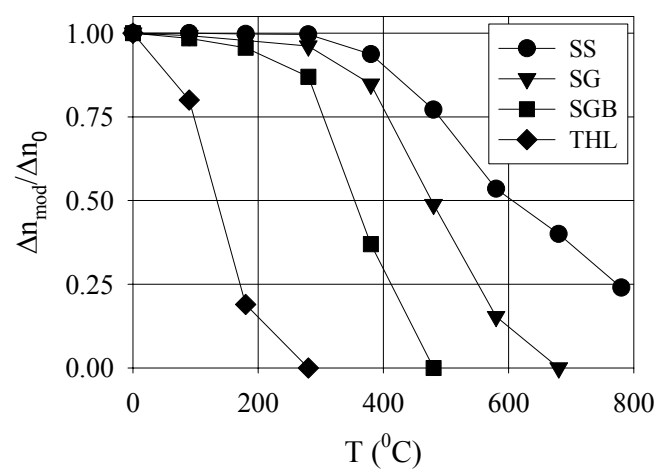

Fig. 1. Temperature stability of gratings written in tin-silicate (SS), germanosilicate (SG), boro-germanosilicate (SGB) and hydrogen loaded telecom (THL) fibers. $\Delta \mathrm{n}_{\text {mod }}$ and $\Delta \mathrm{n}_{0}$ represents the refractive index modulations at temperature $\mathrm{T}$ and 20 ${ }^{\circ} \mathrm{C}$ respectively. 
Fig. 1 shows the temperature behavior of the grating reflectivity and clearly demonstrates the enhanced thermal stability of gratings written in SS with respect to the others. Fig. 2 illustrates the temperature dependence of Bragg shift in a SS fiber.

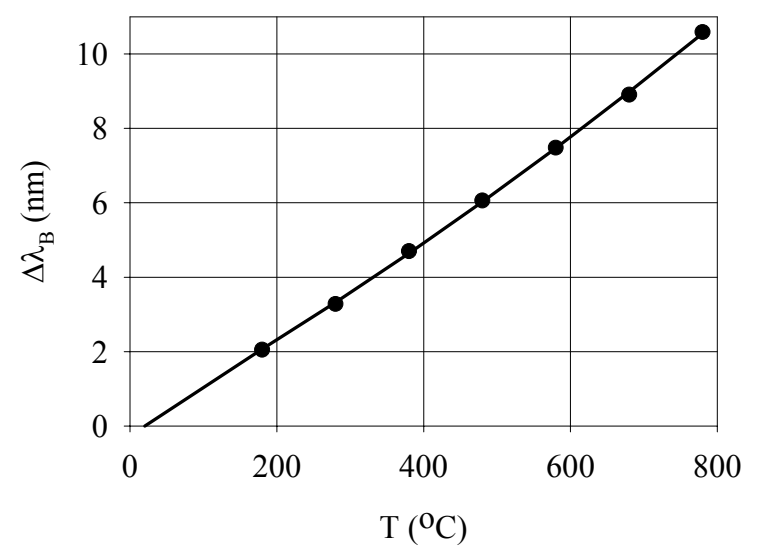

Fig. 2. Dependence of Bragg shift $\Delta \lambda_{\mathrm{B}}$ on temperature T in SS fiber. $\Delta \lambda_{\mathrm{B}}$ was assumed to be 0 at $20^{\circ} \mathrm{C}$.

While the trend at low temperatures is linear, at high temperatures it is polynomial, probably because of the nonlinear temperature dependence of the silica expansion coefficient. Finally, the repetition of thermal cycle for each temperature shows excellent repeatability in the Bragg shifts. In particular, at high temperatures Bragg shift reproducibility is better than $2 \%$.

\section{Conclusions}

The comparison with traditional fibers has shown the enhanced thermal stability of gratings written in the tin doped fibers. Due to the improved stability above $500{ }^{\circ} \mathrm{C}$ and excellent repeatability, SS fiber is the best choice for high temperature thermometers or fiber Bragg sensors.

\section{References}

[1] K.A.Wickersheim and W.D.Hyatt, "Commercial applications of fiber optic temperature measurement", Fiber Optic Sensors IV, SPIE 1267, p. 84, (1990).

[2] Z.Zhang, K.T.V.Grattan and A.W.Palmer, "Sensitive fibre optic thermometer using Cr:LiSAF fluorescence for bio-medical sensing applications", Proceedings of the 8th International Conference on Optical Fibre Sensors, Monterey, pp. 93-96, (1992).

[3] V.Fernicola and L.Crovini, "A high-temperature digital fiber-optic thermometer", Proceedings of the 10th International Conference on Optical Fibre Sensors, Glasgow, pp. 211-214, (1994).

[4] Z.Zhang, K.T.V.Grattan, A.W.Palmer and B.T. Meggitt, "Thulium-doped intrinsic fiber optic sensor for high temperature measurements $\left(>1100{ }^{\circ} \mathrm{C}\right)$ ”, Rev. Scient. Instr. 69, 3210-3214, (1998)

[5] W.W.Morey and G.Meltz, "High temperature capabilities and limitations of fiber grating sensors", Proceedings of the 10th International Conference on Optical Fibre Sensors, Glasgow, pp. 234-237, (1994).

[6] G. Brambilla, V. Pruneri and L. Reekie, "Photorefractive index gratings in SnO2:SiO2 optical fibers", Appl. Phys. Lett. 76, 807-809 (2000). 